\title{
Novel proteins associated with risk for coronary heart disease or stroke among postmenopausal women identified by in-depth plasma proteome profiling
}

Ross L Prentice ${ }^{1 *}$, Sophie J Paczesny ${ }^{2}$, Aaron Aragaki ${ }^{1}$, Lynn M Amon ${ }^{1}$, Lin Chen ${ }^{1}$, Sharon J Pitteri ${ }^{1}$, Martin Mclntosh ${ }^{1}$, Pei Wang ${ }^{1}$, Tina Buson Busald ${ }^{1}$, Judith Hsia ${ }^{3}$, Rebecca D Jackson ${ }^{4}$, Jacques E Rossouw ${ }^{5}$, JoAnn E Manson ${ }^{6}$, Karen Johnson ${ }^{7}$, Charles Eaton', Samir M Hanash ${ }^{1}$

\begin{abstract}
Background: Coronary heart disease (CHD) and stroke were key outcomes in the Women's Health Initiative (WHI) randomized trials of postmenopausal estrogen and estrogen plus progestin therapy. We recently reported a large number of changes in blood protein concentrations in the first year following randomization in these trials using an in-depth quantitative proteomics approach. However, even though many affected proteins are in pathways relevant to the observed clinical effects, the relationships of these proteins to CHD and stroke risk among postmenopausal women remains substantially unknown.

Methods: The same in-depth proteomics platform was applied to plasma samples, obtained at enrollment in the WHI Observational Study, from 800 women who developed CHD and 800 women who developed stroke during cohort follow-up, and from 1-1 matched controls. A plasma pooling strategy, followed by extensive fractionation prior to mass spectrometry, was used to identify proteins related to disease incidence, and the overlap of these proteins with those affected by hormone therapy was examined. Replication studies, using enzyme-linkedimmunosorbent assay (ELISA), were carried out in the WHI hormone therapy trial cohorts.

Results: Case versus control concentration differences were suggested for 37 proteins (nominal $P<0.05$ ) for CHD, with three proteins, beta-2 microglobulin (B2M), alpha-1-acid glycoprotein 1 (ORM1), and insulin-like growth factor binding protein acid labile subunit (IGFALS) having a false discovery rate $<0.05$. Corresponding numbers for stroke were 47 proteins with nominal $P<0.05$, three of which, apolipoprotein A-Il precursor (APOA2), peptidyl-prolyl isomerase A (PPIA), and insulin-like growth factor binding protein 4 (IGFBP4), have a false discovery rate $<0.05$. Other proteins involved in insulin-like growth factor signaling were also highly ranked. The associations of B2M with CHD $(P<0.001)$ and IGFBP4 with stroke $(P=0.005)$ were confirmed using ELISA in replication studies, and changes in these proteins following the initiation of hormone therapy use were shown to have potential to help explain hormone therapy effects on those diseases.
\end{abstract}

Conclusions: In-depth proteomic discovery analysis of prediagnostic plasma samples identified B2M and IGFBP4 as risk markers for CHD and stroke respectively, and provided a number of candidate markers of disease risk and candidate mediators of hormone therapy effects on CHD and stroke.

Clinical Trials Registration: ClinicalTrials.gov identifier: NCT00000611

\footnotetext{
* Correspondence: rprentic@fhcrc.org

1 Division of Public Health Sciences, Fred Hutchinson Cancer Research Center,

1100 Fairview Ave N., Seattle, WA 98102, USA
} 


\section{Background}

Blood protein concentrations provide a source for novel disease risk markers that may be modifiable by treatments or other exposures. As such, protein markers have potential to enhance the understanding of disease pathogenesis, and to elucidate biological processes whereby an exposure affects disease risk.

We report here on a large-scale proteomic study that aimed to uncover novel associations between plasma proteins and the risk of subsequent coronary heart disease (CHD) or stroke. These diseases were key outcomes in Women's Health Initiative (WHI) randomized postmenopausal hormone therapy trials of $0.625 \mathrm{mg} / \mathrm{d}$ conjugated equine estrogen (E-alone), or this same preparation plus $2.5 \mathrm{mg} / \mathrm{d}$ medroxyprogesterone acetate $(\mathrm{E}+\mathrm{P})$. We also sought to identify proteins that both distinguished cases from controls and were altered by $\mathrm{E}$-alone or $\mathrm{E}+\mathrm{P}$ as candidate biomarkers for elucidation of hormone therapy effects on these diseases [1-6]. E-alone and $\mathrm{E}+\mathrm{P}$ were each found to yield an elevation in stroke risk $[3,4]$, whereas $\mathrm{E}+\mathrm{P}$ effects were unfavorable, and unfavorable compared to E-alone effects, for CHD [5,6]. A related research effort is considering case versus control comparisons for breast cancer $[7,8]$.

We recently reported blood proteomic changes between baseline and 1 year for 50 women assigned to active treatment in each of the E-alone and $\mathrm{E}+\mathrm{P}$ trials $[9,10]$. An intact protein analysis system (IPAS) [11-14] was used for these analyses. Under stringent criteria for protein identification and relative quantification, 378 proteins were quantified [10]. There was some evidence (nominal $P<0.05$ ) of change from baseline to 1 year with either or both of $\mathrm{E}$-alone and $\mathrm{E}+\mathrm{P}$ for a remarkable $44.7 \%$ of these proteins. These proteins were involved in coagulation, inflammation, immune response, metabolism, cell adhesion, growth factors, and osteogenesis; pathways that plausibly relate to observed clinical effects [1-8] for these regimens.

A comparatively larger number of study subjects is needed to detect modest associations between plasma proteins and subsequent risk of CHD or stroke. Hence, we contrasted pools formed by equal plasma volumes from 100 cases or from 100 pair-matched controls, with eight such pool pairs for each of the study diseases. We report here on proteins, and sets of proteins, having evidence of a case-control difference in plasma concentration for CHD or stroke, and on the overlap of these proteins with those altered by Ealone or E+P. Enzyme-linked-immunosorbent assay (ELISA) replication studies in the WHI hormone therapy trial cohorts were carried out subsequently for selected proteins.

\section{Methods}

Study subjects and outcome ascertainment

Cases and controls were drawn from the WHI observational study, a prospective cohort study of 93,676 postmenopausal women in the age range 50 to 79 years at enrollment during 1993 to 1998 [15,16]. Fasting blood specimens were obtained at baseline as a part of eligibility screening. Serum and plasma samples were shipped to a central repository and stored at $-70^{\circ} \mathrm{C}$. Disease events during cohort follow-up were initially selfreported, followed by physician adjudication at participating WHI clinical centers, and central adjudication of some outcomes [17]. CHD was composed of myocardial infarction and death due to coronary disease. Cases of hospitalized stroke were based on rapid neurologic deficit attributable to obstruction or rupture of the arterial system or on a demonstrable lesion compatible with acute stroke. CHD and stroke cases were chosen as the earliest 800 incident cases during cohort follow-up for which a suitable plasma specimen was available. Each case was 1-1 matched to a control woman who did not develop any of the study diseases during cohort followup. Cases and controls were matched on baseline age (within 1 year), self-reported ethnicity, hysterectomy status, prior history of the study disease, and enrollment date (median difference 1 month). Non-overlapping sets of controls were chosen for CHD, stroke, and breast cancer. Diagnosis occurred an average of 2.2 and 4.5 years after blood draw for the CHD and stroke cases, respectively.

\section{Sample preparation, protein fractionation, and mass spectrometry analysis}

We used 3,200 patient samples (800 stroke cases, 800 CHD cases, and 1,600 controls) to form case and control pool pairs for 16 IPAS experiments $(8$ stroke +8 CHD). For each IPAS experiment, a case and control pool was created using $5 \mu \mathrm{l}$ of EDTA plasma for each of the 100 cases or 100 controls for proteomic analysis. The pools were independent, with each sample used in only one pool. The IPAS analytic methods used for this project have been described [13] and detailed information is available in Additional file 1. Following immunodepletion of the six most abundant proteins (albumin, IgG, IgA, transferrin, haptoglobin, antitrypsin), pools were concentrated and case and control pools were isotopically labeled with either the 'light' C12 or the 'heavy' C13 acrylamide. The case and corresponding control pools were then mixed together for further analysis.

The combined sample was diluted, and each sample was separated into eight fractions using anion exchange chromatography, and each fraction was further separated using reversed-phase chromatography. 
Lyophilized aliquots from the reversed-phase fractionation were subjected to in-solution trypsin digestion, and individual digested fractions from each reversedphase run were combined, giving a total of $96(8 \times 12$ reversed-phase) fractions for analysis from each original mixed case and control pool. Tryptic peptides were analyzed by a LTQ-FT mass spectrometer. Spectra were acquired in a data-dependent mode in a mass/charge range of 400 to 1,800 , and the 5 most abundant +2 or +3 ions were selected from each spectrum for tandem mass spectrometry (MS/MS) analysis.

\section{Protein identification and case versus control} concentration assessment

The acquired liquid chromatography MS/MS data were processed by a Computational Proteomics Analysis System [18]. Database searches were performed using X! Tandem against the human International Protein Index (IPI) using tryptic search [18]. Database search results were analyzed using PeptideProphet [19] and ProteinProphet [20]. Protein identification was based on ProteinProphet scores that indicate an error rate of less than $10 \%$.

The relative quantification of case versus control concentration for cysteine-containing peptides (acrylamide label binds to cysteine) identified by MS/MS was extracted using a script [11] that calculates the relative peak areas of heavy to light acrylamide-labeled peptides; see [10] for further details. Proteins from all IPAS experiments for a specific disease were aligned by their protein group number, assigned by ProteinProphet, in order to identify master groups of indistinguishable proteins across experiments. Ratios for these protein groups were logarithmically transformed and median-centered at zero for each IPAS experiment. Groups that had fewer than four peptide ratios across all experiments for a specific disease, groups that contained proteins that were targeted for depletion, and groups in which all proteins had been annotated as 'defunct' by IPI, were excluded from analysis.

\section{Statistical analysis of case versus control protein concentrations}

Data analysis was based on $\log$ (base2) concentration ratios from case versus control pools. The log-ratios for a particular protein were analyzed using linear models that included a disease-specific mean parameter plus a variable defined as 1 if the heavy acrylamide label was assigned to the case group and -1 otherwise. A weighted moderated $t$-test [21], implemented in the $\mathrm{R}$ package LIMMA [22], was used to examine whether there was evidence of a disease-specific mean parameter that differs from zero, after adjusting for any labeling effect. The log-ratios were weighted by the number of quantified peptides for each protein. Log-ratios for all three diseases were used to jointly estimate model parameters (the heavy acrylamide label was randomly assigned to the case or control pool for both stroke and breast cancer, and to the case pools for CHD), and to increase the degrees of freedom for log-ratio variance estimation. One of the breast cancer pool pairs gave log-ratios that were comparatively highly variable, and is excluded from all analyses. Benjamini and Hochberg's method [23] was used to accommodate multiple testing, through the calculation of estimated false discovery rates (FDRs), separately for each study disease.

\section{Biological pathway analyses}

A regularized Hotelling $\mathrm{T}^{2}$ procedure was used to identify sets of proteins, defined by biological pathways, that differ in concentrations between cases and controls for each study disease. This testing procedure takes advantage of the correlation structure among the log-ratios for proteins in a given set. Protein sets were defined using the Kyoto Encyclopedia of Genes and Genomes (KEGG) database [24,25].

\section{ELISA replication analyses}

Selected protein associations with disease risk were further evaluated by ELISA testing of CHD and stroke cases and controls drawn from the non-overlapping WHI hormone therapy trial cohorts. Baseline plasma samples were evaluated for women who developed CHD or stroke during the first year following randomization, along with 1-1 matched disease-free controls. Matching variables included age, randomization date, hysterectomy status, and prevalent study disease. Assays were performed according to manufacturer's direction, for beta-2 microglobulin (B2M; Genway San Diego, CA, USA) and insulin-like growth factor binding protein 4 (IGFBP4; R \& D Systems Minneapolis, MN, USA). All samples were assayed with sample characteristics blinded and in duplicate.

\section{Results}

Plasma protein risk markers

Additional file 2 provides information on baseline characteristics for the $800 \mathrm{CHD}$ and 800 stroke cases and their non-overlapping 1-1 matched controls. All women were postmenopausal and in the age range 50 to 79 years at recruitment. Most were white. About two-thirds were overweight or obese. There were few current cigarette smokers. Sixteen percent of CHD cases had experienced a myocardial infarction and $15 \%$ of stroke cases had experienced a stroke prior to WHI enrollment.

Case versus control concentration ratios were determined following application of stringent standards for identification and quantification (see Methods). 
Following application of an additional requirement that proteins were quantified for at least two of the pool pairs for a disease, 346 proteins for CHD and 366 proteins for stroke were included in statistical analyses. Of these, a total of 37 proteins have nominal significance levels of $P<0.05$ for CHD cases versus controls, compared to 17.3 expected by chance; and 47 have $P<0.05$ for stroke cases versus controls, compared to 18.3 expected by chance. These proteins are listed in Tables 1 and 2 along with their mean log-intensity ratios, $P$-values, and FDRs.
Proteins having small FDRs are likely to be associated with disease risk. Three proteins, B2M, alpha-1-acid glycoprotein 1 (ORM1), and insulin-like growth factor binding protein, acid labile subunit (IGFALS) have a FDR $<0.05$ for association with CHD risk; and three proteins, apolipoprotein A-II precursor (APOA2), peptidyl-prolyl isomerase A (PPIA), and IGFBP4 have a FDR $<0.05$ for association with stroke risk. Six other proteins have a FDR $<0.20$ for CHD association, and 14 have a FDR $<0.20$ for stroke association. Figure 1 shows peptide coverage and case versus control concentration

Table 1 Proteins having some evidence $(P<0.05)$ of difference in concentration between coronary heart disease cases and controls

\begin{tabular}{|c|c|c|c|c|}
\hline Protein & Description & Log(base2) case vs control ratio & $P$-value ${ }^{a}$ & $\mathrm{FDR}^{\mathrm{a}}$ \\
\hline B2M & Beta-2-microglobulin. & 0.212 & $5.07 e-05$ & 0.0176 \\
\hline ORM1 & Alpha-1-acid glycoprotein 1 & 0.120 & 0.000182 & 0.0315 \\
\hline IGFALS & Insulin-like growth factor-binding protein complex acid labile chain & -0.112 & 0.000384 & 0.0443 \\
\hline THBS1 & Thrombospondin-1 & -0.632 & 0.00133 & 0.0749 \\
\hline LPA & Apolipoprotein(A) & 0.347 & 0.00138 & 0.0749 \\
\hline CFD & Complement factor D preproprotein & 0.210 & 0.00141 & 0.0749 \\
\hline PRG4 & Isoform C of proteoglycan 4 & 0.232 & 0.00152 & 0.0749 \\
\hline GPX3 & Glutathione peroxidase 3 & -0.224 & 0.00308 & 0.133 \\
\hline IGFBP1 & Insulin-like growth factor-binding protein 1 & 0.423 & 0.00381 & 0.146 \\
\hline MST1 & Hepatocyte growth factor-like protein homolog & -0.306 & 0.00592 & 0.205 \\
\hline $\mathrm{IT} \mid \mathrm{H} 2$ & Inter-alpha-trypsin inhibitor heavy chain $\mathrm{H} 2$ & -0.140 & 0.00786 & 0.247 \\
\hline ENO1 & Isoform alpha-enolase of alpha-enolase & -0.418 & 0.00950 & 0.255 \\
\hline C9 & Complement component C9 & 0.0827 & 0.00989 & 0.255 \\
\hline SFTPB & Pulmonary surfactant-associated protein B precursor & 0.551 & 0.0112 & 0.255 \\
\hline FHL1 & cDNA FL55259 highly similar to four and a half lim domains protein 1 & -0.481 & 0.0116 & 0.255 \\
\hline CRISP3 & cDNA FL75207 & 0.147 & 0.0118 & 0.255 \\
\hline SERPIND1 & Serpin peptidase inhibitor clade D (heparin cofactor) member 1 & 0.210 & 0.0176 & 0.334 \\
\hline CD5L & CD5 antigen-like & 0.152 & 0.0181 & 0.334 \\
\hline SOD3 & Extracellular superoxide dismutase [Cu-Zn] & 0.453 & 0.0183 & 0.334 \\
\hline TPI1 & Triosephosphate isomerase 1 isoform 2 & -0.144 & 0.0232 & 0.401 \\
\hline $\mathrm{C} 1 \mathrm{QB}$ & Complement component $1 \mathrm{Q}$ subcomponent B chain precursor & -0.106 & 0.0271 & 0.407 \\
\hline ATRN & Isoform 1 of attractin & -0.151 & 0.0274 & 0.407 \\
\hline INHBE & Inhibin beta E chain & 0.384 & 0.0284 & 0.407 \\
\hline CHRDL2 & Isoform 2 of chordin-like protein 2 & -0.647 & 0.0287 & 0.407 \\
\hline LIMS1 & cDNA FL55516 highly similar to particularly interesting new Cys-His protein & -0.412 & 0.0318 & 0.407 \\
\hline VASP & Vasodilator-stimulated phosphoprotein & -0.499 & 0.0356 & 0.407 \\
\hline C8A & Complement component C8 alpha chain & 0.170 & 0.0359 & 0.407 \\
\hline C2 & Complement C2 (fragment) & -0.230 & 0.0361 & 0.407 \\
\hline CD14 & Monocyte differentiation antigen CD14 & 0.105 & 0.0361 & 0.407 \\
\hline GC & Vitamin D-binding protein & -0.0451 & 0.0364 & 0.407 \\
\hline MTPN & Myotrophin & -0.240 & 0.0372 & 0.407 \\
\hline SERPINF2 & Serpin peptidase inhibitor, clade F, member 2 & -0.110 & 0.0383 & 0.407 \\
\hline ACTA2 & Actin aortic smooth muscle & -1.22 & 0.0388 & 0.407 \\
\hline TAGLN2 & Transgelin-2 & -0.186 & 0.0426 & 0.433 \\
\hline FERMT3 & Isoform 2 of fermitin family homolog 3 & -0.560 & 0.0462 & 0.454 \\
\hline F12 & Coagulation factor XII & -0.147 & 0.0472 & 0.454 \\
\hline AFM & Afamin & -0.0764 & 0.0490 & 0.458 \\
\hline
\end{tabular}

${ }^{\mathrm{a}} P$-value $=$ significance level for no difference in protein concentration; FDR = estimated false discovery rate. 
Table 2 Proteins having some evidence $(P<0.05)$ of difference in concentration between stroke cases and controls

\begin{tabular}{|c|c|c|c|c|}
\hline Protein & Description & $\begin{array}{l}\text { Log(base2) case vs } \\
\text { control ratio }\end{array}$ & $\begin{array}{c}P- \\
\text { value }^{\mathrm{a}}\end{array}$ & $\mathrm{FDR}^{\mathrm{a}}$ \\
\hline APOA2 & Apolipoprotein A-II & -0.120 & $2.71 e-05$ & 0.00991 \\
\hline PPIA & Peptidyl-prolyl cis-trans isomerase A & 0.194 & $7.68 \mathrm{e}-05$ & 0.0141 \\
\hline IGFBP4 & Insulin-like growth factor-binding protein 4 & 0.409 & 0.000320 & 0.0391 \\
\hline F2 & Prothrombin (fragment) & -0.0732 & 0.000702 & 0.0642 \\
\hline IGF2 & Isoform 1 of insulin-like growth factor II & -0.0694 & 0.00225 & 0.138 \\
\hline C6 & Complement component 6 precursor & -0.140 & 0.00227 & 0.138 \\
\hline LILRA3 & Leukocyte immunoglobulin-like receptor subfamily a member 3 & 0.316 & 0.00341 & 0.177 \\
\hline HPX & Hemopexin & -0.0448 & 0.00407 & 0.177 \\
\hline IGFBP6 & Insulin-like growth factor-binding protein 6 & 0.667 & 0.00435 & 0.177 \\
\hline LOC650157 & Similar to peptidyl-pro cis trans isomerase & 0.237 & 0.00510 & 0.187 \\
\hline IGFBP2 & Insulin-like growth factor-binding protein 2 & 0.480 & 0.00609 & 0.189 \\
\hline GC & Vitamin D-binding protein & -0.0532 & 0.00699 & 0.189 \\
\hline CADM1 & Isoform 1 of cell adhesion molecule 1 & -0.199 & 0.00762 & 0.189 \\
\hline PIN1 & Peptidyl-prolyl cis-trans isomerase NIMA-interacting 1 & 0.190 & 0.00767 & 0.189 \\
\hline CTSD & Cathepsin D & 0.490 & 0.00776 & 0.189 \\
\hline COL1A1 & Collagen alpha-1(I) chain & 0.195 & 0.00826 & 0.189 \\
\hline F13B & Coagulation factor XIII b chain & 0.121 & 0.00903 & 0.194 \\
\hline MANSC1 & MANSC domain-containing protein 1 & -0.962 & 0.0102 & 0.207 \\
\hline COL6A3 & Isoform 1 of collagen alpha-3(VI) chain & 0.828 & 0.0109 & 0.210 \\
\hline GRN & $\begin{array}{l}\text { CDNA FLJ13286 fis clone OVARC1001154 highly similar to Homo sapiens clone } 24720 \\
\text { epithelin } 1 \text { and } 2 \text { mRNA }\end{array}$ & 0.316 & 0.0130 & 0.238 \\
\hline RNASE1 & Ribonuclease pancreatic & 0.582 & 0.0143 & 0.243 \\
\hline MTPN & Myotrophin & 0.249 & 0.0146 & 0.243 \\
\hline GLIPR2 & Golgi-associated plant pathogenesis-related protein 1 & 0.623 & 0.0168 & 0.265 \\
\hline ADAMTSL2 & ADAMTS-like protein 2 & 0.205 & 0.0184 & 0.265 \\
\hline ITIH4 & Isoform 2 of inter-alpha-trypsin inhibitor heavy chain $\mathrm{H} 4$ & -0.238 & 0.0187 & 0.265 \\
\hline HLA-DRB5 ${ }^{\mathrm{b}}$ & Non-secretory ribonuclease & 0.784 & 0.0188 & 0.265 \\
\hline KLKB1 & Plasma kallikrein & -0.115 & 0.0202 & 0.270 \\
\hline CD59 & CD59 glycoprotein & 0.866 & 0.0208 & 0.270 \\
\hline CD14 & Monocyte differentiation antigen CD14 & 0.104 & 0.0214 & 0.270 \\
\hline CSF1R & Macrophage colony-stimulating factor 1 receptor & 0.259 & 0.0223 & 0.272 \\
\hline GRB2 & Isoform 1 of growth factor receptor-bound protein 2 & 1.58 & 0.0235 & 0.278 \\
\hline CD5L & CD5 antigen-like & 0.147 & 0.0253 & 0.289 \\
\hline $\mathrm{B} 2 \mathrm{M}$ & Beta-2-microglobulin & 0.0728 & 0.0280 & 0.310 \\
\hline SERPINC1 & Antithrombin-III & -0.0631 & 0.0312 & 0.325 \\
\hline FCN3 & Isoform 1 of ficolin-3 & 0.132 & 0.0323 & 0.325 \\
\hline HGFAC & Hepatocyte growth factor activator & -0.592 & 0.0324 & 0.325 \\
\hline RBP4 & Retinol-binding protein 4 & 0.0478 & 0.0346 & 0.325 \\
\hline CFHR5 & Complement factor $\mathrm{H}$-related 5 & -0.0800 & 0.0348 & 0.325 \\
\hline PRDX2 & Peroxiredoxin-2 & -0.533 & 0.0361 & 0.325 \\
\hline C8A & Complement component C8 alpha chain & -0.179 & 0.0373 & 0.325 \\
\hline ADAMTSL4 & Isoform 1 of ADAMTS-like protein 4 & -0.130 & 0.0373 & 0.325 \\
\hline QSOX1 & Isoform 1 of sulfhydryl oxidase 1 & 0.370 & 0.0376 & 0.325 \\
\hline CPB2 & Isoform 1 of carboxypeptidase B2 & -0.228 & 0.0381 & 0.325 \\
\hline FETUB & Fetuin-B & 0.0662 & 0.0410 & 0.332 \\
\hline PPIF & Peptidyl-prolyl cis-trans isomerase mitochondrial & 0.318 & 0.0414 & 0.332 \\
\hline LCN2 & Neutrophil gelatinase-associated lipocalin & 0.172 & 0.0417 & 0.332 \\
\hline DSC1 & Isoform 1B of desmocollin-1 & -0.265 & 0.0438 & 0.341 \\
\hline
\end{tabular}

${ }^{\mathrm{a} P \text {-value }}$ = significance level for no difference in protein concentration; FDR = estimated false discovery rate. ${ }^{\mathrm{b}}$ The DRB5 protein group also includes ZNF749, LOC100133811, LOC100133484, LOC100133661, HLA-DRB1, HLA-DRB4, RNASE2, and HLA-DRB3. 


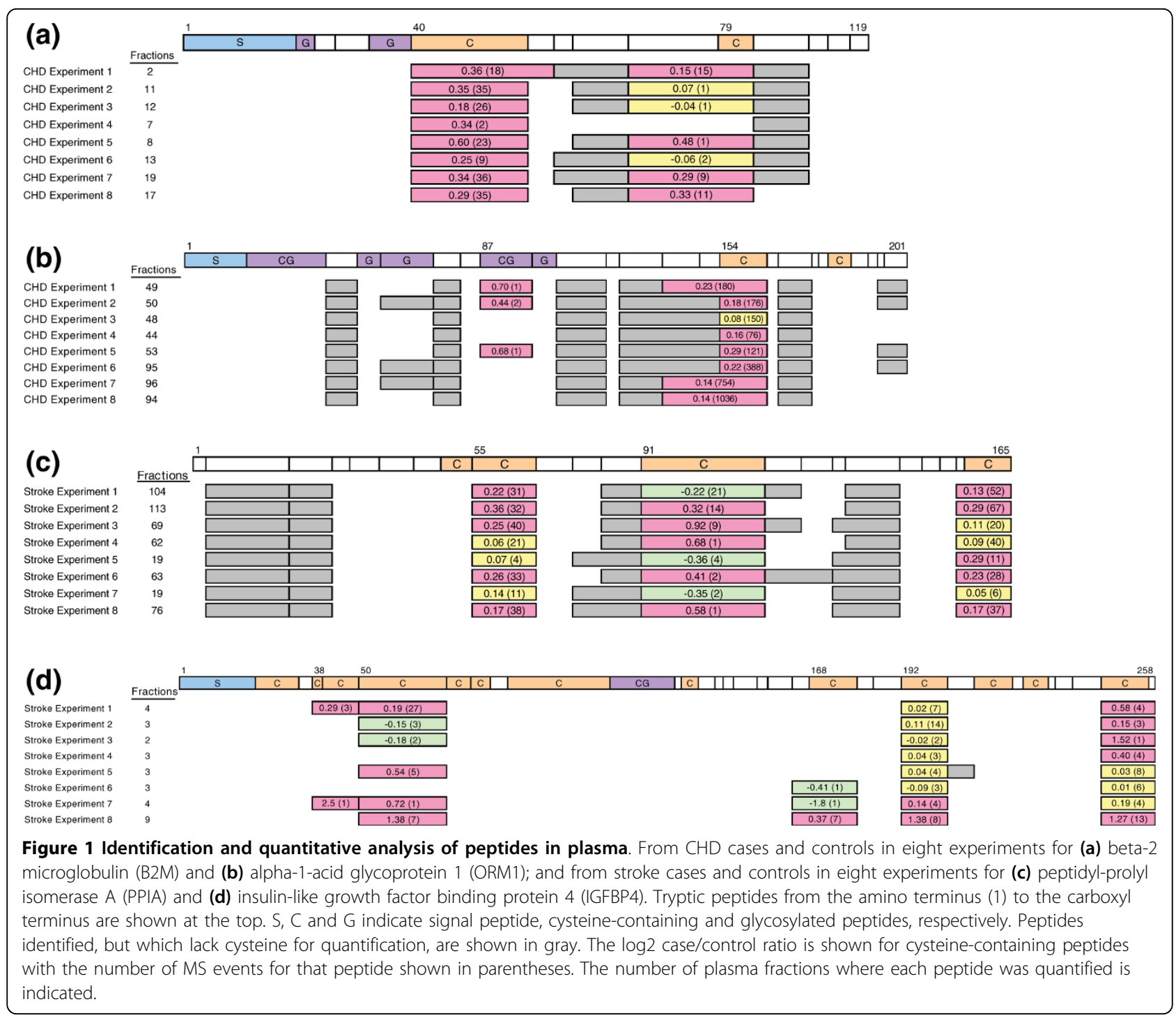

ratios for B2M, ORM1, PPIA, and IGFBP4 separately for each plasma pool pair. Additional files 3 and 4 show $P$-values and FDRs for the entire set of proteins quantified separately for the CHD and stroke analyses. These tables also provide information on the number of peptides and unique peptides identified, and on the number of peptides and unique peptides quantified for each listed protein. IPI numbers corresponding to the gene/ protein are also listed.

\section{Protein levels that are also affected by postmenopausal hormone therapy}

Table 3 shows the subset of Table 1 proteins that appeared to have concentrations affected $(P<0.05)$ by one or both of $\mathrm{E}+\mathrm{P}$ or E-alone in earlier proteomic discovery work [10], while Table 4 provides this information for the corresponding subset of Table 2. Five of the
6 proteins having a FDR $<0.05$ for disease association are influenced by hormone therapy. In addition to these, certain other IGF binding proteins are evidently influenced by hormone therapy and may be related to CHD (IGFBP1) or stroke (IGFBP2, IGFBP6).

\section{Protein set (pathway) analyses}

For each disease, we focused attention on KEGG pathways for which relative quantification was available for three or more proteins and tested for evidence of a case versus control difference in plasma concentrations for the set of quantified proteins. For CHD there were two pathways having $P<0.05$, namely a mitogen-activated protein kinase (MAPK) signaling pathway $(P=0.02)$, which included six quantified proteins (NTRK2, FLNA, CD14, TGFB1, FGFR1, and CACNA2D1), and a glycolysis and gluconeogenesis metabolic pathway $(P=0.03)$, 
Table 3 Proteins having some evidence $(P<0.05)$ of concentration difference between CHD cases and controls that are altered $(P<0.05)$ by postmenopausal hormone therapy

\begin{tabular}{|c|c|c|c|c|c|c|c|c|}
\hline \multirow[b]{2}{*}{ Protein } & \multirow[b]{2}{*}{ Description } & \multicolumn{3}{|c|}{ CHD } & \multicolumn{2}{|l|}{$E+P$} & \multicolumn{2}{|l|}{ E-alone } \\
\hline & & $\begin{array}{l}\text { Log(base2) case } \\
\text { vs control ratio }\end{array}$ & $\begin{array}{c}P- \\
\text { value }^{\mathrm{a}}\end{array}$ & $\mathrm{FDR}^{\mathrm{a}}$ & $\begin{array}{l}\text { Log(base2) case } \\
\text { vs control ratio }\end{array}$ & $\begin{array}{c}P- \\
\text { value }^{\mathrm{a}}\end{array}$ & $\begin{array}{l}\text { Log(base2) case } \\
\text { vs control ratio }\end{array}$ & $\begin{array}{c}P- \\
\text { value }^{a}\end{array}$ \\
\hline B2M & Beta-2-microglobulin & 0.212 & 5.07e-05 & 0.0176 & 0.208 & 0.00205 & 0.230 & 0.00110 \\
\hline IGFALS & $\begin{array}{l}\text { Insulin-like growth factor-binding } \\
\text { protein complex acid labile chain }\end{array}$ & -0.112 & 0.000384 & 0.0443 & 0.151 & 0.00785 & 0.143 & 0.0282 \\
\hline CFD & Complement factor D preproprotein & 0.210 & 0.00141 & 0.0749 & -0.246 & 0.00871 & -0.0472 & 0.620 \\
\hline PRG4 & Isoform C of proteoglycan 4 & 0.232 & 0.00152 & 0.0749 & 0.0735 & 0.181 & 0.128 & 0.0327 \\
\hline |GFBP1 & $\begin{array}{l}\text { Insulin-like growth factor-binding } \\
\text { protein } 1\end{array}$ & 0.423 & 0.00381 & 0.146 & 0.528 & 0.00242 & 1.270 & $3.66 \mathrm{e}-06$ \\
\hline MST1 & $\begin{array}{l}\text { Hepatocyte growth factor-like } \\
\text { protein homolog }\end{array}$ & -0.306 & 0.00592 & 0.205 & 0.530 & 0.0100 & 0.633 & 0.00195 \\
\hline C9 & Complement component C9 & 0.0827 & 0.00989 & 0.255 & 0.101 & 0.0645 & 0.179 & 0.00858 \\
\hline SERPIND1 & $\begin{array}{l}\text { Serpin peptidase inhibitor clade D } \\
\text { (heparin cofactor) member } 1\end{array}$ & 0.210 & 0.0176 & 0.334 & 0.450 & 0.0240 & 0.156 & 0.344 \\
\hline $\mathrm{CIQB}$ & $\begin{array}{l}\text { Complement component } 1 \mathrm{Q} \\
\text { subcomponent B chain precursor }\end{array}$ & -0.106 & 0.0271 & 0.407 & 0.0113 & 0.465 & 0.0480 & 0.0125 \\
\hline ATRN & Isoform 1 of attractin & -0.151 & 0.0274 & 0.407 & -0.190 & 0.000213 & -0.126 & 0.00366 \\
\hline INHBE & Inhibin beta $\mathrm{E}$ chain & 0.384 & 0.0284 & 0.407 & 0.258 & 0.0723 & 0.520 & 0.00734 \\
\hline CHRDL2 & Isoform 2 of chordin-like protein 2 & -0.647 & 0.0287 & 0.407 & -0.301 & 0.0415 & -0.000906 & 0.993 \\
\hline $\mathrm{C} 8 \mathrm{~A}$ & $\begin{array}{l}\text { Complement component C8 alpha } \\
\text { chain }\end{array}$ & 0.170 & 0.0359 & 0.407 & -0.206 & 0.000163 & -0.202 & 0.000121 \\
\hline C2 & Complement C2 (fragment) & -0.230 & 0.0361 & 0.407 & 0.334 & 0.00371 & 0.291 & 0.0107 \\
\hline GC & Vitamin D-binding protein & -0.0451 & 0.0364 & 0.407 & 0.231 & $3.10 \mathrm{e}-06$ & 0.237 & $2.75 e-06$ \\
\hline SERPINF2 & $\begin{array}{l}\text { Serpin peptidase inhibitor, clade } F \text {, } \\
\text { member } 2\end{array}$ & -0.110 & 0.0383 & 0.407 & 0.0922 & 0.148 & 0.166 & 0.0247 \\
\hline $\mathrm{F} 12$ & Coagulation factor XII & -0.147 & 0.0472 & 0.454 & 0.261 & 0.000102 & 0.252 & 0.000219 \\
\hline AFM & Afamin & -0.0764 & 0.0490 & 0.458 & 0.0580 & 0.119 & 0.177 & 0.000330 \\
\hline
\end{tabular}

${ }^{\mathrm{a}} P$-value $=$ significance level for no difference in protein concentration; FDR = estimated false discovery rate.

which included nine quantified proteins (LDHB, LDHA, PKM2, ALDOA, ALDOC, TPI1, GAPDH, ENO1, PGK1). The FDRs were 0.09 for both pathways.

In comparison, there were six pathways having $P<$ 0.05 for stroke; four of which had a FDR $<0.05$. These four were a hematopoietic cell lineage pathway (CD44, GP1BA, C5F1R, CD59, CD14), a purine metabolism pathway (AK1, AK2, PKM2), a peroxisome proliferatoractivated receptor signaling pathway (APOA2, FABP4, FABP1), and a glycolysis and gluconeogenesis pathway having a set of quantified proteins (PKM2, ALDOA, ALDOC, ALDOB, TPI1, ENO2, GAPDH, ENO1, PGK1) that strongly overlaps that listed above for CHD. Figure 2 shows the substantial peptide coverage of glycolytic pathway proteins in the stroke IPAS experiments.

\section{ELISA replication studies}

$\mathrm{B} 2 \mathrm{M}$ is of specific interest for CHD in view of higher levels in cases versus controls, and higher levels following 1-year of use of either $\mathrm{E}+\mathrm{P}$ or E-alone (Table 3). IGFBP4 is of specific interest for stroke for these same reasons (Table $4)$. Hence, these proteins were selected for ELISA replication studies in the WHI hormone therapy trial cohorts.
Based on individual plasma samples from $106 \mathrm{CHD}$ cases occurring during the first year following randomization in the hormone therapy trials, and from 1-1 matched controls, ELISA evaluation yielded B2M concentrations that were $17.9 \%$ higher $(P<0.001)$ in cases versus controls (geometric mean of log-ratios of 1.179 with $95 \%$ confidence interval (CI) of 1.107 to 1.290$)$, very similar to the $15.8 \%\left(2^{0.212}=1.158\right)$ higher concentration in cases compared to controls from the IPAS analyses of Table 1 . Further analysis of case versus control log-ratios, which included the matching variables and several other CHD risk factors to control for possible confounding, produced similar findings (geometric mean of 1.275 with $95 \%$ CI of 1.122 to 1.450 ).

Based on individual plasma samples from 68 stroke cases occurring during the first year following randomization in the hormone therapy trials, and from 1-1 matched controls, ELISA evaluation yielded IGFBP4 concentrations that were $16.6 \%$ higher $(P=0.005)$ in cases versus controls (geometric mean of log-ratios of 1.166 with $95 \%$ CI of 1.050 to 1.295 ). The ELISA case versus control ratio was little altered by additional control for several other potential stroke confounding 
Table 4 Proteins having some evidence $(P<0.05)$ of concentration difference between stroke cases and controls that are altered $(P<0.05)$ by postmenopausal hormone therapy

\begin{tabular}{|c|c|c|c|c|c|c|c|c|}
\hline \multirow[b]{2}{*}{ Protein } & \multirow[b]{2}{*}{ Description } & \multicolumn{3}{|c|}{ Stroke } & \multicolumn{2}{|l|}{$E+P$} & \multicolumn{2}{|l|}{ E-alone } \\
\hline & & $\begin{array}{l}\text { Log(base2) case } \\
\text { vs control ratio }\end{array}$ & $\begin{array}{c}P- \\
\text { value }^{\mathrm{a}}\end{array}$ & $\mathrm{FDR}^{\mathrm{a}}$ & $\begin{array}{l}\text { Log(base2) case } \\
\text { vs control ratio }\end{array}$ & $\begin{array}{c}P- \\
\text { value }^{\mathrm{a}}\end{array}$ & $\begin{array}{l}\text { Log(base2) case } \\
\text { vs control ratio }\end{array}$ & $\begin{array}{c}P- \\
\text { value }^{\mathrm{a}}\end{array}$ \\
\hline APOA2 & Apolipoprotein A-II & -0.120 & $2.71 e-05$ & 0.00991 & 0.212 & 0.000532 & 0.302 & $1.75 \mathrm{e}-05$ \\
\hline PPIA & $\begin{array}{l}\text { Peptidyl-prolyl cis-trans isomerase } \\
\text { A }\end{array}$ & 0.194 & $7.68 \mathrm{e}-05$ & 0.0141 & 0.381 & 0.00899 & 0.201 & 0.126 \\
\hline IGFBP4 & $\begin{array}{l}\text { Insulin-like growth factor-binding } \\
\text { protein } 4\end{array}$ & 0.409 & 0.000320 & 0.0391 & 0.179 & 0.102 & 0.511 & 0.000697 \\
\hline F2 & Prothrombin (fragment) & -0.0732 & 0.000702 & 0.0642 & 0.0633 & 0.00366 & 0.0282 & 0.138 \\
\hline C6 & $\begin{array}{l}\text { Complement component } 6 \\
\text { precursor }\end{array}$ & -0.140 & 0.00227 & 0.138 & -0.123 & 0.00151 & -0.171 & 0.000123 \\
\hline LILRA3 & $\begin{array}{l}\text { Leukocyte immunoglobulin-like } \\
\text { receptor subfamily A member } 3\end{array}$ & 0.316 & 0.00341 & 0.177 & -0.237 & 0.00874 & -0.281 & 0.000277 \\
\hline HPX & Hemopexin & -0.0448 & 0.00407 & 0.177 & 0.123 & $6.65 e-05$ & 0.117 & 0.000124 \\
\hline IGFBP6 & $\begin{array}{l}\text { Insulin-like growth factor-binding } \\
\text { protein } 6\end{array}$ & 0.667 & 0.00435 & 0.177 & 0.0868 & 0.235 & 0.207 & 0.0158 \\
\hline IGFBP2 & $\begin{array}{l}\text { Insulin-like growth factor-binding } \\
\text { protein } 2\end{array}$ & 0.480 & 0.00609 & 0.189 & -0.420 & 0.00477 & -0.287 & 0.0317 \\
\hline GC & Vitamin D-binding protein & -0.0532 & 0.00699 & 0.189 & 0.231 & $3.10 \mathrm{e}-06$ & 0.237 & $2.75 e-06$ \\
\hline CADM1 & $\begin{array}{l}\text { Isoform } 1 \text { of cell adhesion } \\
\text { molecule } 1\end{array}$ & -0.199 & 0.00762 & 0.189 & -0.0139 & 0.875 & 0.180 & 0.0249 \\
\hline COL1A1 & Collagen alpha-1(I) chain & 0.195 & 0.00826 & 0.189 & -0.896 & $5.40 \mathrm{e}-07$ & -0.575 & $8.80 \mathrm{e}-05$ \\
\hline COL6A3 & $\begin{array}{l}\text { Isoform } 1 \text { of collagen alpha-3(VI) } \\
\text { chain }\end{array}$ & 0.828 & 0.0109 & 0.210 & -0.197 & 0.00852 & -0.0134 & 0.834 \\
\hline RNASE1 & Ribonuclease pancreatic & 0.582 & 0.0143 & 0.243 & 0.0346 & 0.311 & 0.0953 & 0.0427 \\
\hline ITIH4 & $\begin{array}{l}\text { Isoform } 2 \text { of inter-alpha-trypsin } \\
\text { inhibitor heavy chain } \mathrm{H} 4\end{array}$ & -0.238 & 0.0187 & 0.265 & 0.458 & 0.000733 & 0.374 & 0.00495 \\
\hline KLKB1 & Plasma kallikrein & -0.115 & 0.0202 & 0.270 & 0.252 & 0.00208 & 0.230 & 0.00187 \\
\hline B2M & Beta-2-microglobulin & 0.0728 & 0.0280 & 0.310 & 0.208 & 0.00205 & 0.230 & 0.00110 \\
\hline SERPINC1 & Antithrombin-III & -0.0631 & 0.0312 & 0.325 & -0.196 & $5.05 e-06$ & -0.143 & $5.50 \mathrm{e}-05$ \\
\hline FCN3 & Isoform 1 of ficolin-3 & 0.132 & 0.0323 & 0.325 & 0.0351 & 0.0287 & 0.0357 & 0.0333 \\
\hline HGFAC & Hepatocyte growth factor activator & -0.592 & 0.0324 & 0.325 & -0.191 & 0.0979 & -0.308 & 0.00765 \\
\hline RBP4 & Retinol-binding protein 4 & 0.0478 & 0.0346 & 0.325 & 0.167 & 0.000117 & 0.177 & 0.000262 \\
\hline CFHR5 & Complement factor $\mathrm{H}$-related 5 & -0.0800 & 0.0348 & 0.325 & 0.179 & 0.000264 & 0.241 & $2.76 \mathrm{e}-05$ \\
\hline PRDX2 & Peroxiredoxin-2 & -0.533 & 0.0361 & 0.325 & 0.691 & 0.0201 & -0.0266 & 0.925 \\
\hline $\mathrm{C} 8 \mathrm{~A}$ & $\begin{array}{l}\text { Complement component C8 alpha } \\
\text { chain }\end{array}$ & -0.179 & 0.0373 & 0.325 & -0.206 & 0.000163 & -0.202 & 0.000121 \\
\hline FETUB & Fetuin-B & 0.0662 & 0.0410 & 0.332 & 0.783 & $1.09 \mathrm{e}-09$ & 0.741 & $1.02 \mathrm{e}-09$ \\
\hline
\end{tabular}

${ }^{\mathrm{a}} P$-value = significance level for no difference in protein concentration; FDR = estimated false discovery rate.

factors (geometric mean of 1.149 with $95 \%$ CI of 1.008 to 1.309 following this control).

Figure 3 shows the B2M assessments for individual CHD cases and controls and the IGFBP4 assessments for individual stroke cases and controls in these replication studies.

\section{Discussion}

The proteomic discovery and replication studies presented here show plasma B2M to be a risk marker for $\mathrm{CHD}$ in postmenopausal women. B2M is an amyloidogenic protein that is elevated in hemodialysis patients and in patients having bone disease [26,27]. B2M has been reported to be associated with CHD risk factors, and an inverse association with HDL cholesterol [28]. Positive associations with peripheral arterial disease [29] and with total mortality among elderly Japanese men and women [30] have also been reported.

Our finding of B2M elevation in plasma obtained months or years prior to CHD diagnosis appears to be novel. Logistic regression analysis of ELISA B2M data yield odds ratios (95\% CI) for the second, third, and fourth quartile of B2M, compared to the first, of 1.28 $(0.46,3.53), 1.77(0.63,4.96)$, and 3.40 (1.23, 9.35), with a trend test having $P=0.002$, in analyses that control for case-control matching factors as well as hormone therapy randomization assignment, hysterectomy status, ethnicity, and history of myocardial infarction. From 


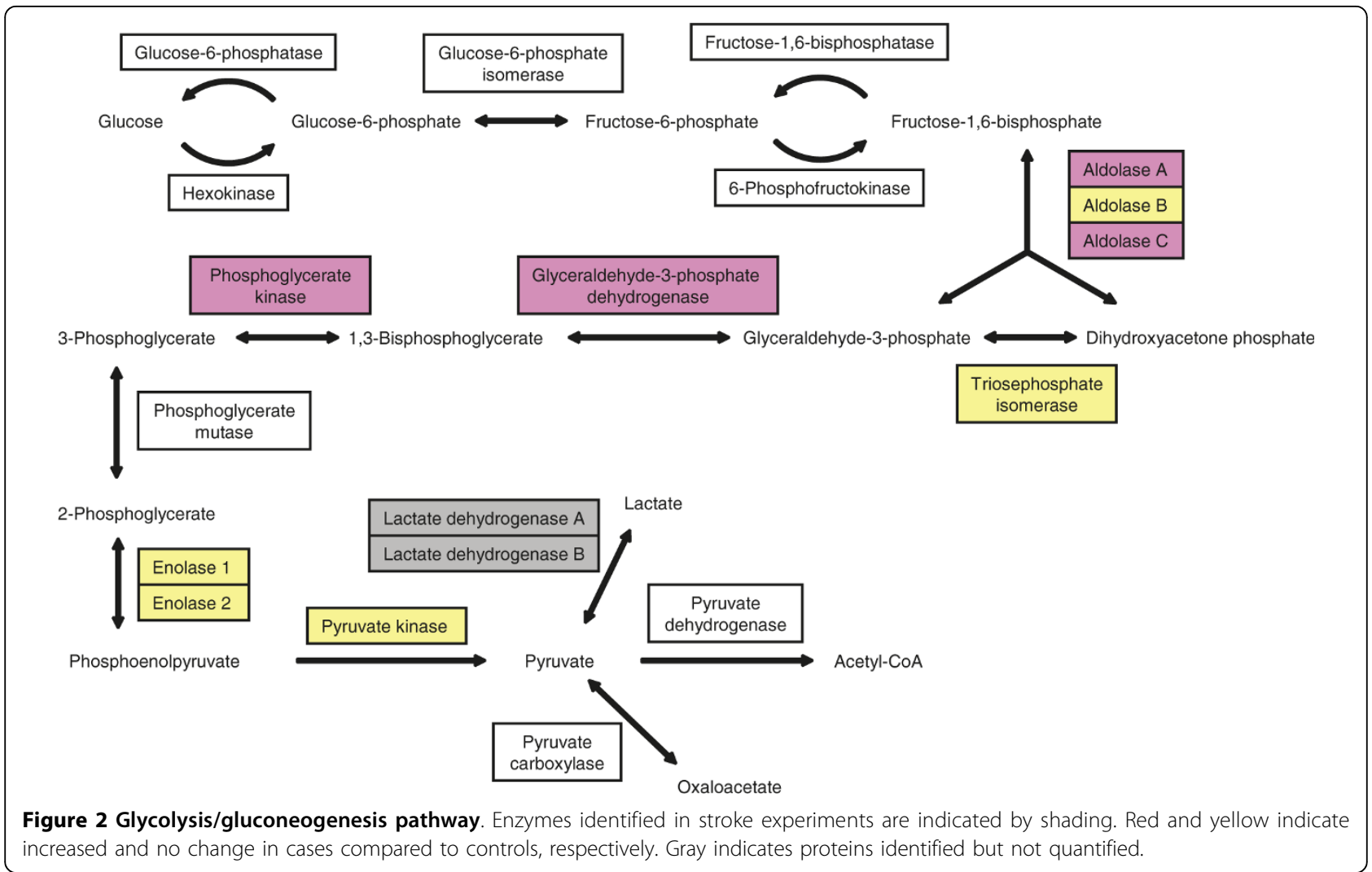

Table 3 we see that B2M levels increased by an estimated $15.5 \%\left(2^{0.208}=1.155\right)$ following $\mathrm{E}+\mathrm{P}$ use and by $17.3 \%\left(2^{0.230}=1.173\right)$ following E-alone use. A $16 \%$ elevation in $\mathrm{B} 2 \mathrm{M}$ projects a $\mathrm{CHD}$ odds ratio $(95 \% \mathrm{CI})$ of $1.30(1.11,1.54)$ based on a logistic regression analysis with a linear term in $\log \mathrm{B} 2 \mathrm{M}$, as determined by ELISA, and these same confounding control variables. Hence, the B2M elevation resulting from hormone therapy use could contribute importantly to an explanation for observed early elevations in CHD risk. The fact that CHD elevations evidently dissipate with longer-term hormone therapy use $[5,6]$ could, for example, reflect concurrent favorable changes in plasma cholesterol fractions, especially for E-alone.

Our proteomic discovery work also suggests (Table 4; $P=0.03$ ) higher B2M levels in stroke cases versus controls, so that this marker may help to understand adverse effects of hormone therapy on cardiovascular disease more generally. The B2M we identified in prediagnostic plasma samples likely differs from modified forms in non-osteotendinous fibrils or insoluble cardiac deposits [31]. However, B2M may provide a valuable focus for studies of disease mechanism and therapeutic intervention in spite of uncertainties about the relationship of plasma levels and pathophysiologic effects within tissue.
The discovery and replication studies presented here also show IGFBP4 to be a risk marker for stroke in postmenopausal women, which appears to be a novel finding. Logistic regression analyses that include a linear term in log IGFBP4 along with the case-control matching variables, hormone therapy randomization assignment, systolic and diastolic blood pressure, body mass index, and indicator variables for cigarette smoking, diabetes, and prior hormone therapy use yield a $P$-value of 0.018 for an association of IGFBP4 with stroke risk. A $20 \%$ increase in IGFBP4, as is consistent with the effects of $\mathrm{E}$-alone and $\mathrm{E}+\mathrm{P}$ on IGFBP4, projects an odds ratio $(95 \% \mathrm{CI})$ of $1.40(1.06,1.85)$ in these analyses, suggesting that this marker could contribute importantly to a mechanistic explanation for the approximate $40 \%$ higher incidence of stroke among $\mathrm{E}$-alone and $\mathrm{E}+\mathrm{P}$ users in the WHI randomized trial $[3,4]$. Also, it is interesting that four of the eleven top-ranked proteins for association with stroke risk (Table 2) are members of the IGF signaling pathway (IGFBP4, IGF2, IGFBP6, IGFBP2). There have been some previous reports of associations between IGF pathway proteins and stroke [32-34]. Increased IGF binding protein levels may result in decreased IGF protein concentrations. IGF1 has been proposed as a potential neuroprotective protein for stroke [35]. 


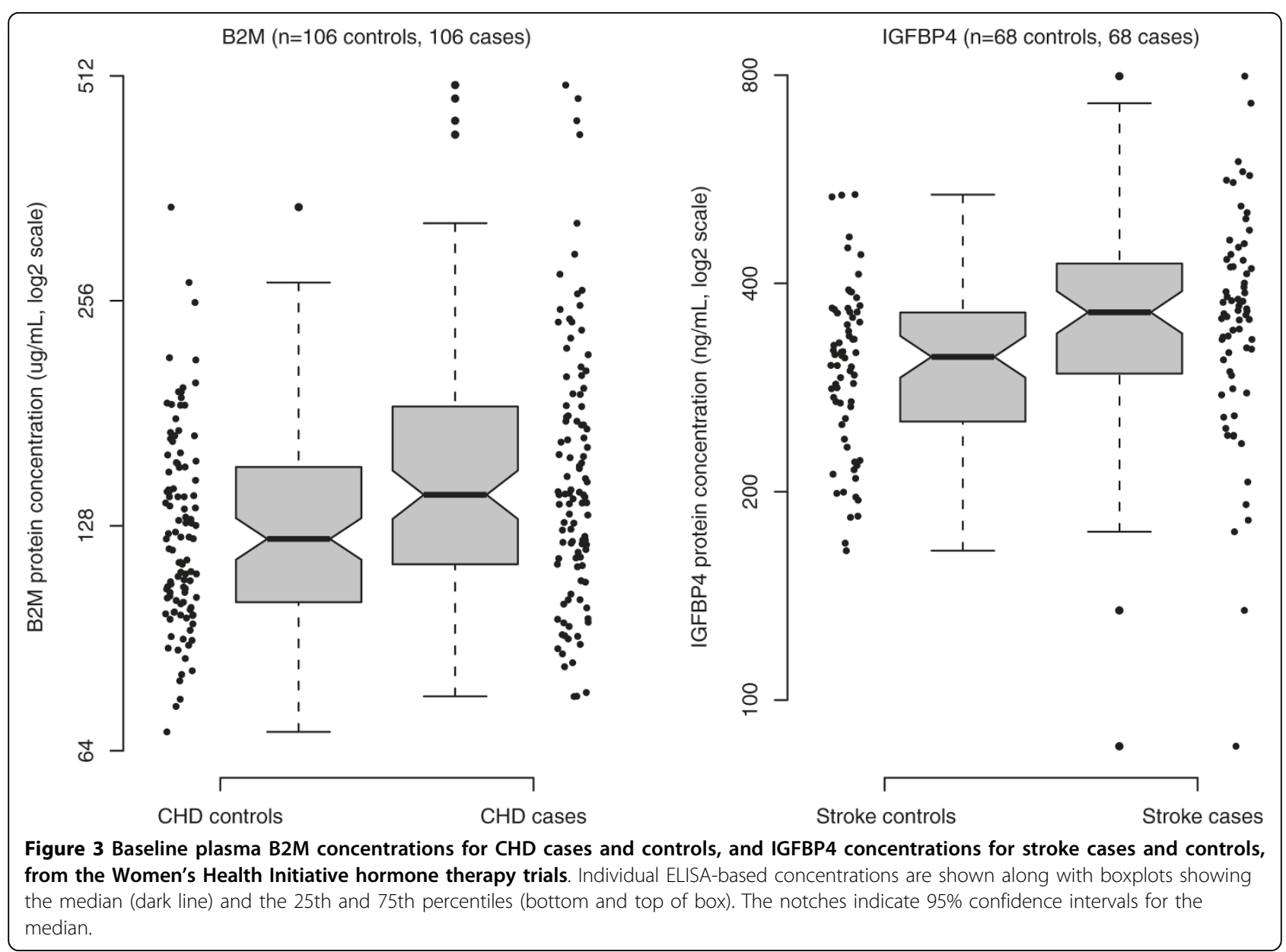

To more directly assess the role of B2M and IGFBP4 in mediating hormone therapy effects on CHD and stroke, respectively, we are currently carrying out ELISA analyses of baseline and 1-year plasma samples in the WHI hormone therapy trials. The effect of changes between baseline and 1-year on these proteins on subsequent hormone therapy hazard ratios for CHD and stroke will be examined.

Other proteins having small FDRs for association with CHD (Table 1) or stroke (Table 2) will benefit from evaluation in replication studies. Some of these have previously received some consideration as vascular disease risk markers, including ORM1 [36-40], APOA2 [41-43], PPIA [44], and IGFALS [45-47].

In addition to protein set analyses based on KEGG pathways (described in Results), we also examined Gene Ontology [48] pathways related to inflammation. There was some evidence $(P=0.03)$ for a difference between CHD cases and controls for a cytokine activity pathway (CCL5, C5, PF4, and CCL16), and some $(P=0.04)$ for an acute inflammatory response pathway (ORM1, ORM2, C2, CFHR1, MBL2, AHSG), whereas there was no evidence of corresponding differences between stroke cases and controls.

\section{Conclusions}

We have identified B2M and IGFBP4 as novel risk markers for CHD and stroke, respectively. These markers have potential to help elucidate hormone therapy effects on these diseases as observed in the WHI randomized controlled trials. The IPAS platform [11-14] provides quantification only for proteins having cysteine residues, but otherwise our analyses benefit from the depth of the proteomic profiling. Concentration ratios associated with hormone therapy in our earlier IPAS studies agreed closely with ELISA-based ratios from the same samples [9], and IPAS concentration ratios for E-alone and E+P agreed closely with each other for many proteins identified as hormone-therapy related. These comparisons suggest that a number of additional proteins with small FDRs (for example, < 0.2) in Tables 1 and 2 are likely also to be disease risk markers, though it will be important for these associations to be replicated in independent samples. 


\section{Additional material}

Additional file 1: Supplementary methods. Detailed methods for sample preparation, protein fractionation, and mass spectrometry analysis are described.

Additional file 2: Table S1. Baseline characteristics for women developing coronary heart disease (CHD) or stroke and for corresponding disease-free controls, drawn from the Women's Health Initiative Observational Study.

Additional file 3: Table S2. CHD case versus control log-transformed concentration ratios for all quantified proteins.

Additional file 4: Table S3. Stroke case versus control log(base2)transformed concentration ratios for all quantified proteins.

\begin{abstract}
Abbreviations
APOA2: apolipoprotein A-II precursor; B2M: beta-2 microglobulin; CHD: coronary heart disease; $\mathrm{Cl}$ : confidence interval; $\mathrm{E}$-alone: estrogen-alone; $\mathrm{E}+\mathrm{P}$. estrogen plus progestin; ELISA: enzyme-linked immunosorbent assay; FDR: false discovery rate; IGFALS: insulin-like growth factor-binding protein acid labile subunit; IGFBP4: insulin-like growth factor binding protein 4; IPAS intact protein analysis system; IPI: International Protein Index; KEGG: Kyoto Encyclopedia of Genes and Genomes; MS/MS: tandem mass spectrometry; ORM1: alpha-1-acid glycoprotein 1; PPIA: peptidyl-prolyl isomerase A; WHI: Women's Health Initiative.
\end{abstract}

\section{Acknowledgements}

The WHI program is funded by the National Heart, Lung, and Blood Institute, National Institutes of Health, US Department of Health and Human Services through contracts N01WH22110, 24152, 32100-2, 32105-6, 32108-9, 32111-13, $32115,32118-, 19,32122,42107-26,42129-32$, and 44221), and particularly by BAA contract \#HHSN268200764315C. Dr Prentice's work was partially supported by grant P01 CA53996 from the National Cancer Institute. Decisions concerning study design, data collection and analysis, interpretation of the results, the preparation of the manuscript, or the decision to submit the manuscript for publication resided with committees composed of $\mathrm{WHI}$ investigators that included $\mathrm{NHLBI}$ representatives. The authors thank the $\mathrm{WHI}$ investigators and staff for their outstanding dedication and commitment. A list of key investigators involved in this research follows. A full listing of $\mathrm{WHI}$ investigators can be found at [49]. Program Office: (National Heart, Lung, and Blood Institute, Bethesda, MD) Elizabeth Nabel, Jacques Rossouw, Shari Ludlam, Linda Pottern, Joan McGowan, Leslie Ford, and Nancy Geller. Clinical Coordinating Center: (Fred Hutchinson Cancer Research Center, Seattle, WA) Ross Prentice, Garnet Anderson, Andrea LaCroix, Charles L Kooperberg, Ruth E Patterson, Anne McTiernan; (Wake Forest University School of Medicine, Winston-Salem, NC) Sally Shumaker; (Medical Research Labs, Highland Heights, KY) Evan Stein; (University of California at San Francisco, San Francisco, CA) Steven Cummings. Clinical Centers: (Albert Einstein College of Medicine, Bronx, NY) Sylvia Wassertheil-Smoller; (Baylor College of Medicine, Houston, TX) Aleksandar Rajkovic; (Brigham and Women's Hospital, Harvard Medical School, Boston, MA) JoAnn Manson; (Brown University, Providence, RI) Annlouise R Assaf; (Emory University, Atlanta, GA) Lawrence Phillips; (Fred Hutchinson Cancer Research Center, Seattle, WA) Shirley Beresford; (George Washington University Medical Center, Washington, DC) Judith Hsia; (Los Angeles Biomedical Research Institute at Harbor-UCLA Medical Center, Torrance, CA) Rowan Chlebowski; (Kaiser Permanente Center for Health Research, Portland, OR) Evelyn Whitlock; (Kaiser Permanente Division of Research, Oakland, CA) Bette Caan; (Medical College of Wisconsin, Milwaukee, WI) Jane Morley Kotchen; (MedStar Research Institute/Howard University, Washington, DC) Barbara V Howard; (Northwestern University, Chicago/Evanston, IL) Linda Van Horn; (Rush Medical Center, Chicago, IL) Henry Black; (Stanford Prevention Research Center, Stanford, CA) Marcia L Stefanick; (State University of New York at Stony Brook, Stony Brook, NY) Dorothy Lane; (The Ohio State University, Columbus, OH) Rebecca Jackson; (University of Alabama at Birmingham, Birmingham, AL) Cora E Lewis; (University of Arizona, Tucson/Phoenix, AZ) Tamsen Bassford; (University at Buffalo, Buffalo, NY) Jean Wactawski-Wende; (University of California at Davis,
Sacramento, CA) John Robbins; (University of California at Irvine, CA) F Allan Hubbell; (University of California at Los Angeles, Los Angeles, CA) Howard Judd; (University of California at San Diego, LaJolla/Chula Vista, CA) Robert D Langer; (University of Cincinnati, Cincinnati, $\mathrm{OH}$ ) Margery Gass; (University of Florida, Gainesville/Jacksonville, FL) Marian Limacher; (University of Hawaii, Honolulu, HI) David Curb; (University of lowa, lowa City/Davenport, IA) Robert Wallace; (University of Massachusetts/Fallon Clinic, Worcester, MA) Judith Ockene; (University of Medicine and Dentistry of New Jersey, Newark, NJ) Norman Lasser; (University of Miami, Miami, FL) Mary Jo O'Sullivan; (University of Minnesota, Minneapolis, MN) Karen Margolis; (University of Nevada, Reno, NV) Robert Brunner; (University of North Carolina, Chapel Hill, NC) Gerardo Heiss; (University of Pittsburgh, Pittsburgh, PA) Lewis Kuller; (University of Tennessee, Memphis, TN) Karen C Johnson; (University of Texas Health Science Center, San Antonio, TX) Robert Brzyski; (University of Wisconsin, Madison, WI) Gloria E Sarto; (Wake Forest University School of Medicine, Winston-Salem, NC) Denise Bonds; (Wayne State University School of Medicine/Hutzel Hospital, Detroit, MI) Susan Hendrix.

\section{Author details}

'Division of Public Health Sciences, Fred Hutchinson Cancer Research Center, 1100 Fairview Ave N., Seattle, WA 98102, USA. ²Department of Pediatrics, University of Michigan Comprehensive Cancer Center, 1500 East Medical Center Drive, Ann Arbor, Ml 48109, USA. ${ }^{3}$ Research and Development, AstraZeneca LP, 1971 Rockland Road, Wilmington, DE 19803, USA. ${ }^{4}$ Division of Endocrinology, Ohio State University, 198 McCampbell, 1581 Dodd Drive, Columbus, OH 43210, USA. ${ }^{5}$ WHI Project Office, National Heart, Lung, and Blood Institute, National Institutes of Health, 6701 Rockledge Drive, Bethesda, MD 20892, USA. 'Division of Preventive Medicine, Brigham and Women's Hospital, Harvard Medical School, 75 Francis Street, Boston, MA 02115, USA. ${ }^{7}$ Department of Preventive Medicine, University of Tennessee Health Sciences Center, 66 N. Pauline, Memphis, TN 38163, USA. ${ }^{8}$ Brown University, Memorial Hospital of Rhode Island, 111 Brewster Street, Pawtucket, RI 02860, USA.

\section{Authors' contributions}

RLP, LMA, LC, SJP (FHCRC), JH, RDJ, JER, JEM, CE, and SMH participated in drafting the manuscript. Data were collected, analyzed, and interpreted by RLP, SJP (University of Michigan), LMA, SJP (FHCRC), MM, TBB, KJ, and SMH. RLP and SMH were responsible for study design. Statistical analysis was performed by AA, LC, MM, PW, and RLP.

\section{Competing interests}

The authors declare that they have no competing interests.

Received: 25 January 2010 Revised: 25 June 2010

Accepted: 28 July 2010 Published: 28 July 2010

\section{References}

1. Women's Health Initiative Steering Committee: Effects of conjugated equine estrogen in postmenopausal women with hysterectomy: the Women's Health Initiative randomized controlled trial. JAMA 2004, 291:1701-1712.

2. Writing Group for the Women's Health Initiative Investigators: Risks and benefits of estrogen plus progestin in healthy postmenopausal women: principal results from the Women's Health Initiative randomized controlled trial. JAMA 2002, 288:321-333.

3. Hendrix SL, Wassertheil-Smoller S, Johnson KC, Howard BV, Kooperberg C, Rossouw JE, Trevisan M, Aragaki A, Baird AE, Bray PF, Buring JE, Criqui MH, Herrington D, Lynch JK, Rapp SR, Torner J, for the WHI Investigators: Effects of conjugated equine estrogen on stroke in the Women's Health Initiative. Circulation 2006, 113:2425-2434.

4. Wassertheil-Smoller S, Hendrix SL, Limacher M, Heiss G, Kooperberg C, Baird A, Kotchen T, Curb JD, Black H, Rossouw JE, Aragaki A, Safford M, Stein E, Laowattana S, Mysiw WJ, for the WHI Investigators: Effect of estrogen plus progestin on stroke in postmenopausal women: the Women's Health Initiative: a randomized trial. JAMA 2003, 289:2673-2684.

5. Hsia J, Langer RD, Manson JE, Kuller L, Johnson KC, Hendrix SL, Pettinger M, Heckbert SR, Greep N, Crawford S, Eaton CB, Kostis JB, Caralis P, Prentice R, for the Women's Health Initiative Investigators: Conjugated equine estrogens and coronary heart disease: the Women's Health Initiative. Arch Intern Med 2006, 166:357-365. 
6. Manson JE, Hsia J, Johnson KC, Rossouw JE, Assaf AR, Lasser NL, Trevisan M, Black HR, Heckbert SR, Detrano R, Strickland OL, Wong ND, Crouse JR, Stein E, Cushman M, for the Women's Health Initiative Investigators: Estrogen plus progestin and the risk of coronary heart disease. $N$ Engl J Med 2003, 349:523-534.

7. Chlebowski RT, Hendrix SL, Langer RD, Stefanick ML, Gass M, Lane D, Rodabough RJ, Gilligan MA, Cyr MG, Thomson CA, Khandekar J, Petrovitch H, McTiernan A, for the WHI Investigators: Influence of estrogen plus progestin on breast cancer and mammography in healthy postmenopausal women: the Women's Health Initiative Randomized Trial. JAMA 2003, 289:3243-3253.

8. Stefanick ML, Anderson GL, Margolis KL, Hendrix SL, Rodabough RJ, Paskett ED, Lane DS, Hubbell FA, Assaf AR, Sarto GE, Schenken RS, Yasmeen S, Lessin L, Chlebowski RT, for the WHI Investigators: Effects of conjugated equine estrogens on breast cancer and mammography screening in postmenopausal women with hysterectomy. JAMA 2006 295:1647-1657.

9. Katayama H, Paczesny S, Prentice $R$, Aragaki A, Faca VM, Pitteri SJ, Zhang Q, Wang H, Silva M, Kennedy J, Rossouw J, Jackson R, Hsia J, Chlebowski R, Manson JE, Hanash SM: Application of serum proteomics to the Women's Health Initiative conjugated equine estrogens trial reveals a multitude of effects relevant to clinical findings. Genome Med 2009, 1:47.

10. Pitteri SJ, Hanash SM, Aragaki A, Amon LM, Chen L, Busald Buson T, Paczesny S, Katayama H, Wang H, Johnson MM, Zhang Q, Mclntosh M, Wang P, Kooperberg C, Rossouw JE, Jackson R, Manson JE, Hsia J, Liu S, Martin L, Prentice RL: Postmenopausal estrogen and progestin effects on the serum proteome. Genome Med 2009, 1:121.

11. Faca V, Coram M, Phanstiel D, Glukhova V, Zhang Q, Fitzgibbon M, McIntosh M, Hanash S: Quantitative analysis of acrylamide labeled serum proteins by LC-MS/MS. J Proteome Res 2006, 5:2009-2018.

12. Faca V, Pitteri SJ, Newcomb L, Glukhova V, Phanstiel D, Krasnoselsky A, Zhang Q, Struthers J, Wang H, Eng J, Fitzgibbon M, McIntosh M, Hanash S: Contribution of protein fractionation to depth of analysis of the serum and plasma proteomes. J Proteome Res 2007, 6:3558-3565.

13. Faca VM, Song KS, Wang H, Zhang Q, Krasnoselsky AL, Newcomb LF, Plentz RR, Gurumurthy S, Redston MS, Pitteri SJ, Pereira-Faca SR, Ireton RC, Katayama H, Glukhova V, Phanstiel D, Brenner DE, Anderson MA, Misek D, Scholler N, Urban ND, Barnett MJ, Edelstein C, Goodman GE,

Thornquist MD, McIntosh MW, DePinho RA, Bardeesy N, Hanash SM: A mouse to human search for plasma proteome changes associated with pancreatic tumor development. PLoS Med 2008, 5:e123.

14. Hanash SM, Pitteri SJ, Faca VM: Mining the plasma proteome for cancer biomarkers. Nature 2008, 452:571-579.

15. The Women's Health Initiative Study Group: Design of the Women's Health Initiative clinical trial and observational study. Control Clin Trials 1998, 19:61-109.

16. Langer RD, White E, Lewis CE, Kotchen JM, Hendrix SL, Trevisan M: The Women's Health Initiative observational study: baseline characteristics of participants and reliability of baseline measures. Ann Epidemio/ 2003, 13(9 Suppl):S107-121.

17. Curb JD, McTiernan A, Heckbert SR, Kooperberg C, Stanford J, Nevitt M, Johnson KC, Proulx-Burns L, Pastore L, Criqui M, Daugherty S, WHI Morbidity and Mortality Committee: Outcomes ascertainment and adjudication methods in the Women's Health Initiative. Ann Epidemiol 2003, 13(9 Suppl):S122-128.

18. Rauch $A$, Bellew $M$, Eng J, Fitzgibbon $M$, Holzman $T$, Hussey $P$, Igra $M$ Maclean B, Lin CW, Detter A, Fang R, Faca V, Gafken P, Zhang $H$, Whiteaker J, States D, Hanash S, Paulovich A, McIntosh MW: Computational Proteomics Analysis System (CPAS): an extensible, open-source analytic system for evaluating and publishing proteomic data and high throughput biological experiments. J Proteome Res 2006, 5:112-121.

19. Keller A, Nesvizhskii Al, Kolker E, Aebersold R: Empirical statistical model to estimate the accuracy of peptide identifications made by MS/MS and database search. Anal Chem 2002, 74:5383-5392.

20. Nesvizhskii Al, Keller A, Kolker E, Aebersold R: A statistical model for identifying proteins by tandem mass spectrometry. Anal Chem 2003, 75:4646-4658.

21. Smyth GK: Linear models and empirical Bayes methods for assessing differential expression in microarray experiments. Stat Appl Genet Mol Biol 2004, 3, Article3.
22. Smyth GK: Limma: linear models for microarray data. Bioinformatics and Computational Biology Solutions using $R$ and Bioconductor New York: SpringerGentleman R, Carey V, Dudoit S, Irizarry R, Huber W 2005, 397-420.

23. Benjamini $Y$, Hochberg $Y$ : Controlling the false discovery rate: a practical and powerful approach to multiple testing. J Roy Stat Soc B (Methodological) 1995, 57:289-300.

24. Kanehisa M: The KEGG database. Novartis Found Symp 2002, 247:91-101, discussion 101-103, 119-128, 244-252.

25. The KEGG PATHWAY Database. [http://www.genome.jp/kegg/pathway html].

26. Gorevic PD, Casey TT, Stone WJ, DiRaimondo CR, Prelli FC, Frangione B: Beta-2 microglobulin is an amyloidogenic protein in man. $J$ Clin Invest 1985, 76:2425-2429.

27. Polat H, Yeksan M, Dalmaz M, Kaptanoglu B, Koşar A, Akkuş I: Serum amyloid A protein levels in haemodialysis patients. Nephrol Dial Transplant 1996, 11:1492-1493.

28. Saijo Y, Utsugi M, Yoshioka E, Horikawa N, Sato T, Gong Y, Kishi R: Relationship of B2-Microglobulin to arterial stiffness in Japanese subjects. Hypertens Res 2005, 28:505-511.

29. Wilson AM, Kimura E, Harada RK, Nair N, Narasimhan B, Meng X-Y, Zhang F, Beck KR, Olin JW, Fung ET, Cooke JP: B2-Microglobulin as a biomarker in peripheral arterial disease: proteomic profiling and clinical studies. Circulation 2007, 116:1396-1403.

30. Shinkai S, Chaves PHM, Fujiwara Y, Watanabe S, Shibata H, Yoshida H, Suzuki T: $B_{2}$-Microglobulin for risk stratification of total mortality in the elderly population. Comparison with Cystatin $\mathrm{C}$ and C-reactive protein. Arch Intern Med 2008, 168:200-206.

31. Stoppini M, Mangione $P$, Monti M, Giorgetti $S$, Marchese $L$, Arcidiaco $P$, Verga L, Segagni S, Pucci P, Merlini G, Bellotti V: Proteomics of beta2microglobulin amyloid fibrils. Biochim Biophys Acta 2005, 1753:23-33.

32. Johnsen SP, Hundborg HH, Sørensen HT, Orskov H, Tjønneland A, Overvad K, Jørgensen JO: Insulin-like growth factor (IGF) I, -II, and IGF binding protein-3 and risk of ischemic stroke. J Clin Endocrinol Metab 2005, 90:5937-5941.

33. Denti L, Annoni V, Cattadori E, Salvagnini MA, Visioli S, Merli MF, Corradi F, Ceresini G, Valenti G, Hoffman AR, Ceda GP: Insulin-like growth factor 1 as a predictor of ischemic stroke outcome in the elderly. Am J Med 2004, 117:312-317.

34. Bondanelli M, Ambrosio MR, Onofri A, Bergonzoni A, Lavezzi S, Zatelli MC, Valle D, Basaglia N, degli Uberti EC: Predictive value of circulating insulinlike growth factor I levels in ischemic stroke outcome. J Clin Endocrinol Metab 2006, 91:3928-3934.

35. Kooijman R, Sarre S, Michotte Y, De Keyser J: Insulin-like growth factor I: a potential neuroprotective compound for the treatment of acute ischemic stroke? Stroke 2009, 40:e83-e88.

36. Fournier T, Medjoubi-N N, Porquet D: Alpha-1-acid glycoprotein. Biochim Biophys Acta 2000, 1482:157-171.

37. Giardina EG, Raby K, Freilich D, Vita J, Brem R, Louie M: Time course of alpha-1-acid glycoprotein and its relation to myocardial enzymes after acute myocardial infarction. Am J Cardiol 1985, 56:262-265.

38. Mori T, Sasaki J, Kawaguchi H, Handa K, Takada Y, Matsunaga A, Kono S, Arakawa K: Serum glycoproteins and severity of coronary atherosclerosis. Am Heart J 1995, 129:234-238.

39. Held C, Hjemdahl P, Håkan Wallén N, Björkander I, Forslund L, Wiman B, Rehnqvist N: Inflammatory and hemostatic markers in relation to cardiovascular prognosis in patients with stable angina pectoris. Results from the APSIS study. The Angina Prognosis Study in Stockholm. Atherosclerosis 2000, 148:179-188.

40. Kuller LH, Tracy RP, Shaten J, Meilahn EN: Relation of C-reactive protein and coronary heart disease in the MRFIT nested case-control study. Multiple Risk Factor Intervention Trial. Am J Epidemiol 1996, 144:537-547.

41. Birjmohun RS, Dallinga-Thie GM, Kuivenhoven JA, Stroes ES, Otvos JD, Wareham NJ, Luben R, Kastelein JJ, Khaw KT, Boekholdt SM: Apolipoprotein A-II is inversely associated with risk of future coronary artery disease. Circulation 2007, 116:2029-2035.

42. Matsuda M, Miyahara T, Murai A, Fujimoto N, Kameyama M: Lipoprotein abnormalities in survivors of cerebral infarction with a special reference to apolipoproteins and triglyceride-rich lipoproteins. Atherosclerosis 1987, 68:131-136. 
43. Walldius G, Aastveit AH, Jungner I: Stroke mortality and the apoB/apoA-I ratio: results of the AMORIS prospective study. J Intern Med 2006, 259:259-266.

44. Satoh K, Nigro P, Matoba T, O'Dell MR, Cui Z, Shi X, Mohan A, Yan C, Abe J, Illig KA, Berk BC: Cyclophilin A enhances vascular oxidative stress and the development of angiotensin II-induced aortic aneurysms. Nat Med 2009, 15:649-656.

45. Colao A: The GH-IGF-I axis and the cardiovascular system: clinical implications. Clin Endocrinol (Oxf) 2008, 69:347-358.

46. Sandhu MS: Insulin-like growth factor-I and risk of type 2 diabetes and coronary heart disease: molecular epidemiology. Endocr Dev 2005, 9:44-54.

47. Boisclair YR, Rhoads RP, Ueki I, Wang J, Ooi GT: The acid-labile subunit (ALS) of the $150 \mathrm{kDa}$ IGF-binding protein complex: an important but forgotten component of the circulating IGF system. J Endocrinol 2001, 170:63-70.

48. Ashburner M, Ball CA, Blake JA, Botstein D, Butler H, Cherry JM, Davis AP, Dolinski K, Dwight SS, Eppig JT, Harris MA, Hill DP, Issel-Tarver L, Kasarskis A, Lewis S, Matese JC, Richardson JE, Ringwald M, Rubin GM, Sherlock G: Gene ontology: tool for the unification of biology. The Gene Ontology Consortium. Nat Genet 2000, 25:25-29.

49. The Women's Health Initiative. [http://www.whi.org].

doi:10.1186/gm169

Cite this article as: Prentice et al: Novel proteins associated with risk for coronary heart disease or stroke among postmenopausal women identified by in-depth plasma proteome profiling. Genome Medicine 2010 2:48.

\section{Submit your next manuscript to BioMed Central and take full advantage of:}

- Convenient online submission

- Thorough peer review

- No space constraints or color figure charges

- Immediate publication on acceptance

- Inclusion in PubMed, CAS, Scopus and Google Scholar

- Research which is freely available for redistribution

Submit your manuscript at www.biomedcentral.com/submit 